\title{
Polymer Surface-Induced Order of Liquid Crystalline Molecular Alignment Based on Nematic-Isotropic Phase Transition Behavior
}

\author{
Sung-Kyu Hong, Hirotsugu KIKUCHI, and Tisato KaJIYAma ${ }^{\dagger}$ \\ Department of Materials Physics and Chemistry, \\ Graduate School of Engineering, Kyushu University, \\ 6-10-1 Hakozaki, Higashiku, Fukuoka 812-8581, Japan
}

(Received August 5, 1998)

\begin{abstract}
Alignment order of liquid crystal (LC) molecules at the (polymer/LC) interface was evaluated by comparing nematic-isotropic phase transition temperatures $\left(T_{\mathrm{NI}}\right)$ at the interface with $T_{\mathrm{NI}}$ in the bulk. The thickness dependence of $T_{\mathrm{NI}}$ was measured using the LC cell in which an LC thin layer was sandwiched between polymer surfaces without surface treatment. The order of LC molecular alignment at the (polymer/LC) interface increased with surface free energy of the polymer substrate. The surface of crystalline polymer provided higher order of LC molecular alignment. Hydrophobic intermolecular interactions at the (polymer/LC) interface may be more responsible for increase in the interfacial molecular alignment order of LC in comparison with the hydrophilic one.
\end{abstract}

KEY WORDS Liquid Crystal/Order Parameter/(Polymer/Liquid Crystal) Interface/ Nematic-Isotropic Phase Transition Temperature / Surface Free Energy /

Substantial research has been devoted to understanding the anchoring mechanisms at the interface between liquid crystalline (LC) molecule and solid substrate. The macroscopic three-dimensional alignment of LC molecules in an LC cell is strongly dependent on two-dimensional LC molecular alignment at the LC-solid substrate interface. LC-polymer substrate interfacial characteristics are inevitably important for making LC display devices, for example, TN (twisted nematic), STN (super twisted nematic) type and so on, because LC molecules fill space between polymer substrates coated on two glass plates. In the case of the (polymer/LC) composite films, the anchoring effect at the (polymer/LC) interface has much influence on the electro-optical switching characteristics, such as the rise or decay response time and contrast between light scattering and transparent states, because LC molecules are bicontinuously embedded in a three-dimensional polymer network. ${ }^{1-3}$ Despite the practical importance for the design and construction of LC display devices, the mechanism of the surface-induced alignment of LC molecules is not well understood.

When LC molecules contact a solid polymer substrate, the order parameter of the LC molecular alignment at the (polymer/LC) interface is not always the same as that in the bulk region, and is strongly dependent on intermolecular interactions with the polymer surface. The interfacial order parameter of LCs on the rubbed poly(vinyl alcohol) (PVA) surface is higher than that in the bulk region. The situation is opposite in the case of the $\mathrm{SiO}$ evaporated surface. Nevertheless, both substrate surfaces give the same homogeneous bulk molecular alignment to $\mathrm{LC}^{4}$

Since no direct method is available to measure the order parameter of LC molecular alignment at the interface, an indirect method on the basis of the Landau-de Gennes theory has been proposed. ${ }^{5-12}$
According to the Landau-de Gennes theory, a nematicisotropic phase transition temperature $\left(T_{\mathrm{NI}}\right)$ at a bulk nematic LC should be dependent on the order parameter of LC molecular alignment. Substrate surface effects on the nematic-isotropic transition of LC have also attracted much attention. Sheng studied the nematic-isotropic transition in a thin layer of nematic LC being held between substrates inducing higher orientational order of LC on the basis of Landau-de Gennes theory. ${ }^{8,9} \mathrm{He}$ found that the $T_{\mathrm{NI}}$ of LC layer increases with the thickness of LC layer, in the case of that the solid surface induces the higher order of LC. Poniewierski and Sluckin applied Sheng's theory to the substrate surface with the lower orientational order of LC. ${ }^{10,11}$ The experimental results above apparently indicate that $T_{\mathrm{NI}}$ of LC layer shifts either upward or downward according to whether the substrate is orientationally ordering or disordering to LC molecules, respectively, following the thermodynamic Kelvin equation. If the substrate is neutral to the alignment order of LC layer, the magnitude of $T_{\mathrm{NI}}$ of $\mathrm{LC}$ at the (polymer/LC) interface remains the same as that in the bulk nematic LC, regardless of the thickness of LC layer. Though it is a valuable method for evaluating the interfacial order of LC to measure the shift of $T_{\mathrm{NI}}$ in the vicinity of the interface, results for polymer surfaces without any surface treatment have not been reported yet.

In this study, the molecular alignment order of LC induced at the (polymer/LC) interface was investigated based on the thickness dependence of the nematic-isotropic phase transition temperature $\left(T_{\mathrm{NI}}\right)$ using the LC cell in which the LC thin layer was sandwiched between polymer surfaces without surface treatment, to clarify the relationship between the interfacial order of LC and polymer surface characteristics.

\footnotetext{
$\uparrow$ To whom correspondence should be addressed.
} 


\section{Polymer}

1 Poly(diisopropyl fumarate) (Pdi-iPF) 6 Polyimide (PI)

2 Poly(1-cyano-1-ethylisopropyl fumarate) 6-1 Solvent soluble PI (S-PI) (PCNEt-iPF)
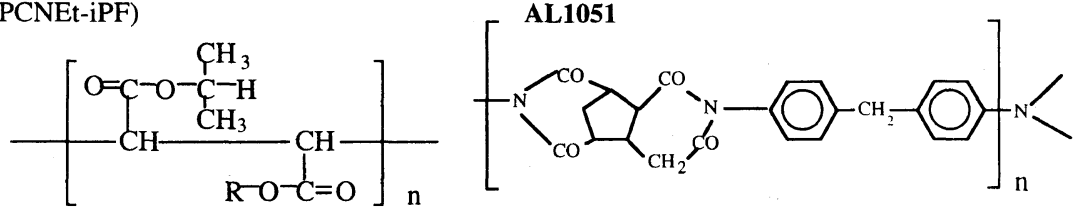

DA-1, DA-2, DA-3
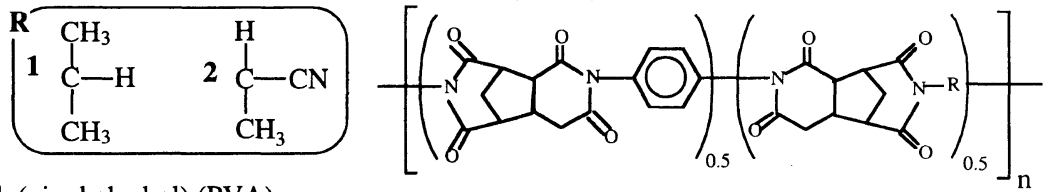

3 Poly(vinyl alcohol) (PVA)<smiles>CC(C)(C)CC(O)C(C)(C)C</smiles>

4 Poly(vinyl chloride) (PVC)<smiles>CC(C)(C)CC(Cl)C(C)(C)C</smiles>

5 Poly(vinylidene floride) (PVDF)<smiles>CC(C)(C)C#CCC(C)(C)C</smiles>

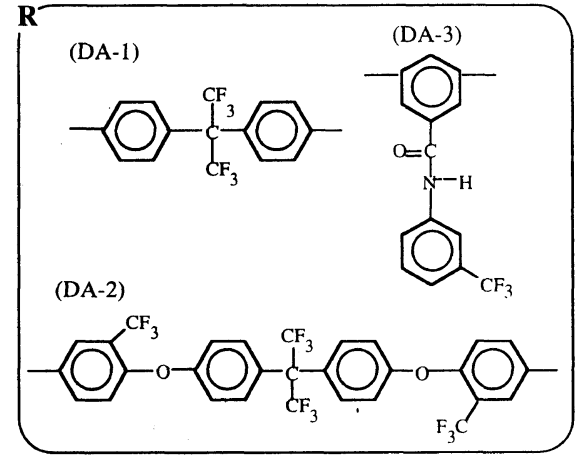

6-2 Thermally polymerized PI (T-PI)

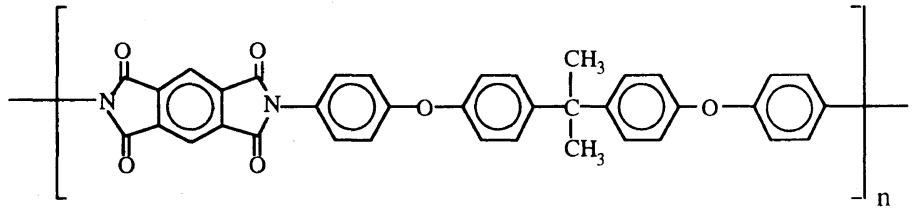

\section{Liquid Crystal}

4-cyano-4'-n-pentylbiphenyl (5CB)

$$
\begin{aligned}
\mathrm{T}_{\mathrm{KN}} & =297 \mathrm{~K} & & \mathrm{~T}_{\mathrm{NI}}=308 \mathrm{~K} \\
\varepsilon_{\perp} & =6.9 & & \varepsilon_{\|}=17.9 \\
\Delta \varepsilon & =11.0 & &
\end{aligned}
$$

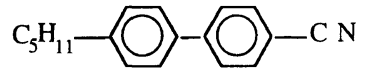

Figure 1. Chemical structures and physical properties of polymers and liquid crystal in this study.

\section{EXPERIMENTAL}

\section{Materials}

Figure 1 shows the chemical structures and physical properties of polymers and liquid crystal used in this study. Poly(diisopropyl fumarate) (Pdi-iPF, Nippon Oil \& Fats Co., Ltd.), poly(1-cyano-1-ethyl-isopropyl fumarate) (PCNEt-iPF, Nippon Oil \& Fats Co., Ltd.), poly(vinyl alcohol) (PVA, Kuraray Co., Ltd.), poly(vinyl chloride) (PVC), poly(vinylidene floride) (PVDF), four types of solvent soluble polyimides (S-PI, JSR Co., Ltd.) with different side chain groups and thermally polymerized polyimide (T-PI, Chisso Co., Ltd.) were used as polymers. 4-Cyano-4'-pentylbiphenyl (5CB, Merck Co., Ltd.) was used as LC. 5CB presents a nematic phase at room temperature.

\section{Measurement of Thickness-Dependent $T_{\mathrm{NI}}$ of $L C$ cell}

Figure 2 shows a cross-sectional view of the LC cell designed to measure the thickness dependence of $T_{\mathrm{NI}}$ of the LC layer. 5CB was sandwiched between optical-flat glass plate and half-convex lens with a $5 \mathrm{~m}$ focal length with both surfaces coated with polymer thin films. The thickness of the LC layer was minimum at the center of the cell and increased continuously along the circumference from the center. Maximum thickness of LC layer at both ends of the optical window was $60 \mu \mathrm{m}$. The cell was surrounded by brass materials to prevent inhomogenous heat transfer at the entire cell as shown in Figure 2. The polymers were dissolved in good solvents, and the both convex lens and flat substrate were spin-coated with their solutions of $1 \mathrm{wt} \%$ under conditions of $4000 \mathrm{rpm}$ and $293 \mathrm{~K}$. $\gamma$-Butyrolactone for four types of S-PIs, pure water for PVA, toluene for Pdi-iPF, chloroform for PCNEt-iPF, cyclohexanone for PVC and $N, N$-dimethylacetamide for PVDF were used as the solvents. After spin-coating, the substrates were annealed at $423 \mathrm{~K}$ for S-PIs, PVA, PVC, PVDF and at $373 \mathrm{~K}$ for Pdi-iPF, PCNEt-iPF for $2 \mathrm{~h}$, respectively. In order to prepare the T-PI film, a solution of poly(amic acid) as a polyimide precursor was spincoated on both convex lens and the flat substrate and then, thermally polymerized in vacuum at $573 \mathrm{~K}$ for $2 \mathrm{~h}$. The thickness of the resulting polymer films on the substrates ranged 


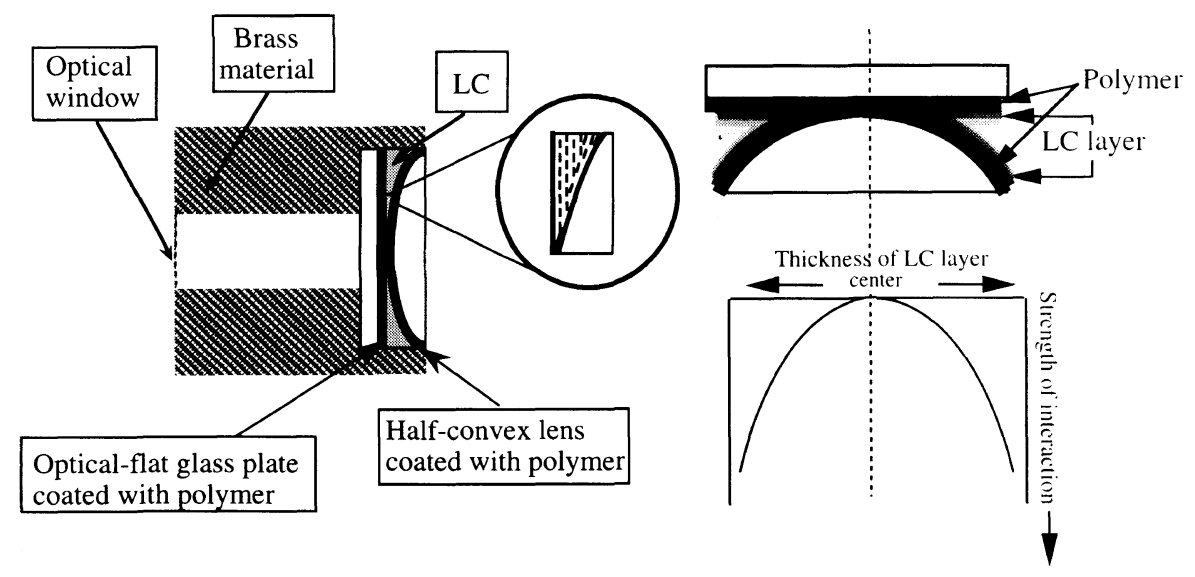

Figure 2. Cross-sectional view of the LC cell designed to measure thickness dependence of $T_{\mathrm{NI}}$ of the LC layer.

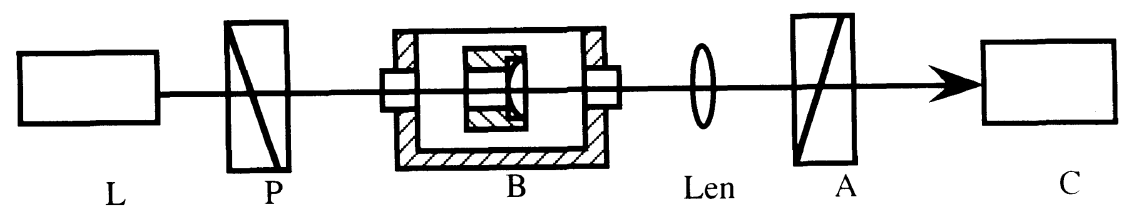

L: light P: polarizer B: constant-temperature bath

Len: lens $\quad$ A: analyzer $\quad$ C: video camera

Figure 3. Schematic representation of experimental set up to measure thickness dependence of $T_{\mathrm{NI}}$ of LC layer.

from sub-micrometer to a few micrometers.

Figure 3 shows a schematic representation of the experimental set up to measure the thickness dependence of $T_{\mathrm{NI}}$ of the LC layer. The thickness dependence of $T_{\mathrm{NI}}$ of the LC layer was measured based on optical-texture changes under crossed nicols upon heating or cooling of the LC cell placed in a constant-temperature bath in which temperature was uniformly controlled over the entire sample. LC layer thickness was estimated from a curvature ratio of lens and the distance from the lens center. White light was used as the light source. Temperature in the thermostat was monitored during observation of change of the optical texture. The precision of temperature measurement should be controlled within $\pm 10^{-3} \mathrm{~K}$ by a quartz thermometer, because shift of $T_{\mathrm{NI}}$ is in the range of $10^{-2}-10^{-3} \mathrm{~K}$. Heating and cooling rates were carefully controlled at $0.01 \mathrm{~K} \mathrm{~min}^{-1}$.

Surface Free Energy and Aggregation Structures of Polymers and Contact Angles of 5CB to Each Polymer Surface

The surface free energy and aggregation structures of polymers and contact angle of 5CB to each polymer surface were measured to investigate their dependence on the order of LC molecular alignment at the (polymer/LC) interface. The surface free energy of each polymer was evaluated from measurement of static contact angle with two solutions such as water and methylene iodide using a Kyowa contact angle meter. Surface free energy was calculated on the basis of Owens equation $^{13}$ and $\gamma_{d}, \gamma_{h}$, and $\gamma_{s}$ corresponding to the dispersion component, the hydrogen bonding component and the sum of the two components, respectively, were obtained. Contact angle measurement of $5 \mathrm{CB}$ with each polymer surface was carried out in an isotropic phase at $313 \mathrm{~K}$. The aggregation structure of each polymer thin film was investigated based on wide angle X-ray diffraction (WAXD) study. WAXD patterns were taken on an imaging plate by using $\mathrm{Ni}$ filtered $\mathrm{Cu}-K_{\alpha}$ $(\lambda=0.15405 \mathrm{~nm})$ radiation from $40 \mathrm{kV}, 300 \mathrm{~mA} \mathrm{X}$-ray source of X-ray generator (Mac Science M18XHF).

\section{RESULTS AND DISCUSSION}

\section{Evaluation of the Order of LC Molecular Alignment at (Polymer/LC) Interface Based on Phase Transition Behavior}

Figure 4 shows the WAXD photographs of each polymer. PVA, PVDF, and T-PI showed strong Debye rings at the Bragg spacings of $0.41-0.48 \mathrm{~nm}$, and thus were crystalline polymers. PVC and DA-1, DA-2, DA-3, and AL1051 were amorphous, because diffuse amorphous halos at Bragg spacings of $0.52-0.58 \mathrm{~nm}$ were observed. For Pdi-iPF and PCNEt-iPF, strong inner Debye rings at the Bragg spacing of $1.08 \mathrm{~nm}$ and $1.15 \mathrm{~nm}$ and diffuse rings at the Bragg spacings of $0.46 \mathrm{~nm}$ and $0.44 \mathrm{~nm}$ were observed. Strong inner Debye rings may be due to regular packing of semi-rigid chains in a similar fashion to LC molecules, owing to the repulsive force between bulky side groups in Pdi-iPF and PCNEt-iPF molecules. ${ }^{14}$ Pdi-iPF and PCNEt-iPF may thus be in an amorphous state with quasi-hexagonal packing of rod-like molecules.

Interfacial interactions affecting the shift of $T_{\mathrm{NI}}$ may extend over $100 \mathrm{~nm}$ from the LC-substrate interface, and continuously decrease with increase in distance from the interface. ${ }^{8}$ Therefore the degree of interfacial interaction can be evaluated from thickness dependence of $T_{\mathrm{NI}}$ of 


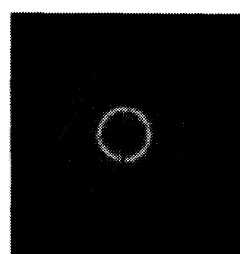

Pdi-iPF

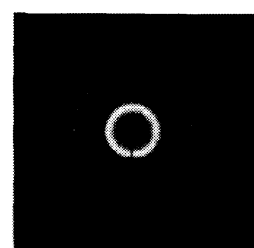

PCNEt-iPF

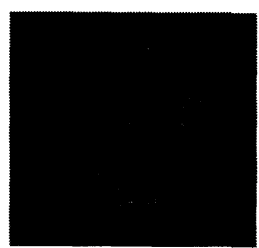

PVC

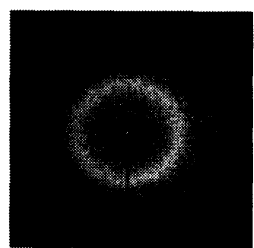

S-PI(DA-1)

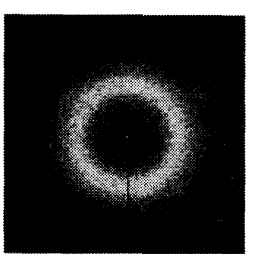

S-PI(DA-2)

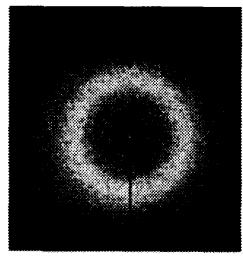

S-PI(DA-3)

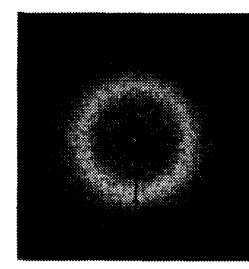

S-PI(AL1051)

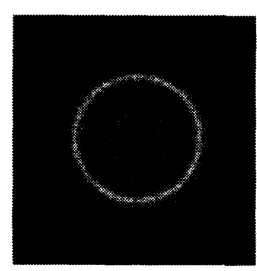

PVA

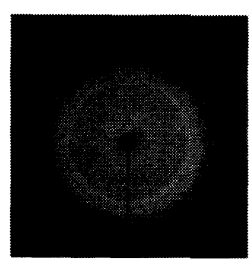

T-PI

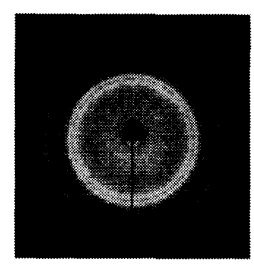

PVDF

Figure 4. WAXD photographs of polymers obtained by WAXD

Nematic phase $\longrightarrow$ Isotropic phase

(Upon heating )

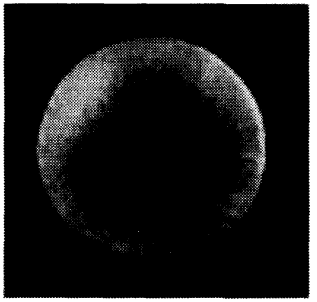

a) $(\mathrm{Pdi}-\mathrm{iPF} / 5 \mathrm{CB})$

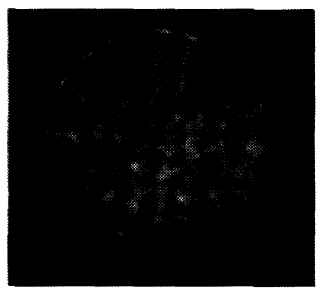

b) $(\mathrm{PCNEt}-\mathrm{iPF} / 5 \mathrm{CB})$

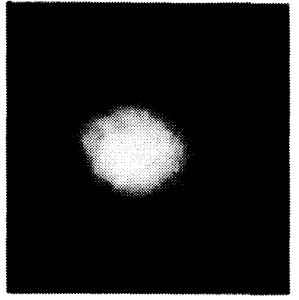

c) $(\mathrm{PVC} / 5 \mathrm{CB}),(\mathrm{PVA} / 5 \mathrm{CB})$ (PVDF/5CB), (PIs/5CB)

Figure 5. Photographs of the LC cell perpendicular to the substrate surface under crossed nicols in the process from the nematic phase to isotropic one upon the heating process.

the LC layer using LC cell as shown in Figure 2, since $T_{\mathrm{NI}}$ of LC should be dependent on the order parameter of LC molecular alignment in a nematic phase.

Figure 5 shows photographs of the LC cell along the direction perpendicular to the substrate surface under crossed nicols in the process from the nematic phase to the isotropic one upon the heating process. The dark and bright regions correspond to isotropic and nematic phases, respectively, because the LC molecular alignments in the nematic state are almost planar to the substrate surface in all cases studied here. In the LC cell, the thickness of LC layer sandwiched between polymer substrates continuously changed from 0 at the center to $60 \mu \mathrm{m}$ at the edge due to the curvature of half-convex lens. It is clear from Figure 5 that nematic-isotropic phase transition upon heating process occurred from the center, or the thinnest part of the 5CB layer sandwiched between Pdi-iPF films, while from the edge, from the thickest part, in the cases of the (PVA/5CB), (PVC) 5CB), (PVDF/5CB), and five (PIs/5CB) systems. For (PCNEt-iPF/5CB) system, the phase transition occurred throughout an observation field.

Figure 6 shows a schematic representation of the optical texture changes for three LC cells shown in Figure
5 during nematic-isotropic phase transition with both heating and cooling. Figures 5 and 6 show that $T_{\mathrm{NI}}$ in the cell is apparently higher at the edge part than the center in the (Pdi-iPF/5CB) system, and $T_{\mathrm{NI}}$ is lower at the edge than center in the (PVA/5CB), (PVC/5CB), (PVDF/5CB), and (PIs/5CB) systems. For (PCNEt$\mathrm{iPF} / 5 \mathrm{CB}$ ) system, $T_{\mathrm{NI}}$ is nearly same throughout the cell. The difference in $T_{\mathrm{NI}}$ between the center and edge of the observation field roughly corresponds to the difference between $T_{\mathrm{NI}}$ at the (polymer/LC) interface and $T_{\mathrm{NI}}$ of the bulk LC, because the surface of polymer substrates coated on the glass plate and convex lens are in contact at the center of the cell $(d \fallingdotseq 0)$ and $d$ at the edge of the cell is quite large, $60 \mu \mathrm{m}$. The experimentally obtained magnitude defined by eq 1 are listed in Table $\mathrm{I}$.

$$
\Delta T_{\mathrm{NI}} \equiv T_{\mathrm{NI}}(\text { center })-T_{\mathrm{NI}}(\text { edge })
$$

$T_{\mathrm{NI}}$ at the (Pdi-iPF/5CB) interface should be lower than that of the bulk, because $\Delta T_{\mathrm{NI}}$ was negative for the LC cell of (Pdi-iPF/5CB). $T_{\mathrm{NI}}$ at the interface should be higher than that of the bulk for (PVC/5CB), (PVDF/ 5CB), (PVA/5CB), and (PIs/5CB) systems because $\Delta T_{\mathrm{NI}} \mathrm{S}$ were positive. From the Sheng, Poniewierski and Sluckin theory, $T_{\mathrm{NI}}$ increases with decrease of thickness of the 


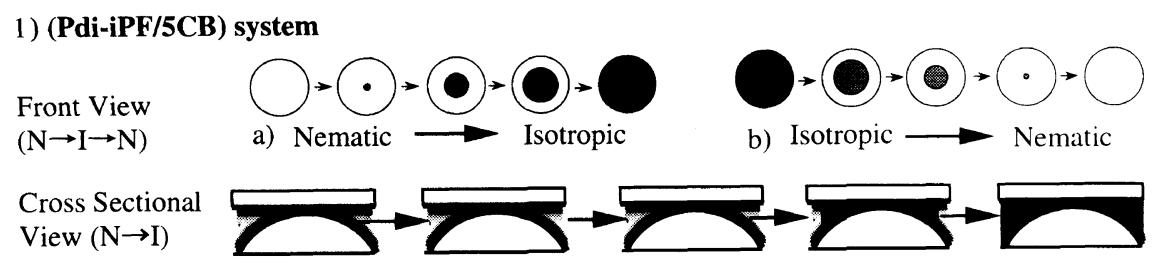

\section{2) (PCNEt-iPF/5CB) system}

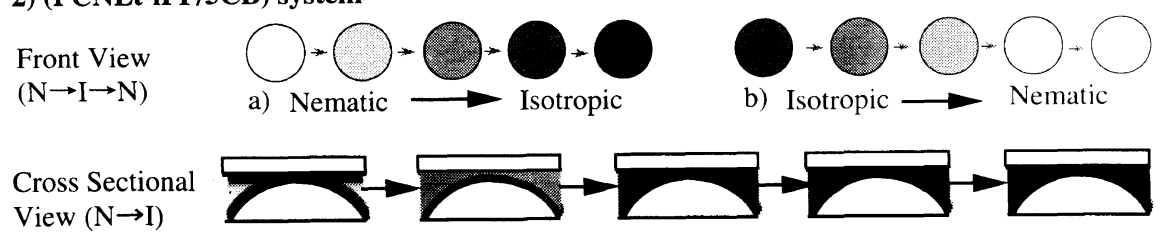

3) (PVC/5CB), (PVA/5CB), (PVDF/5CB) and (PIs/5CB) systems

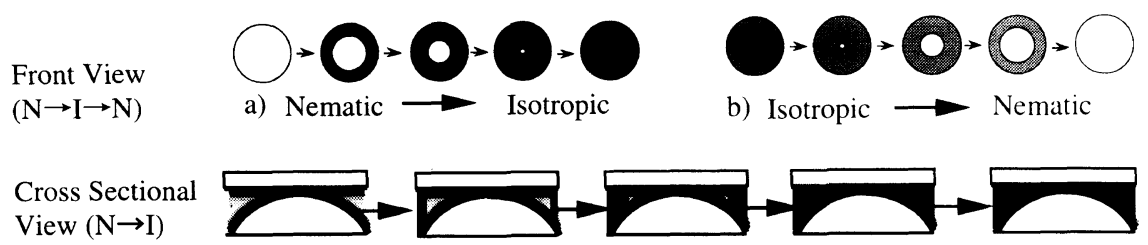

Figure 6. Schematic representation of optical texture changes for the three LC cells during the nematic-isotropic phase transition during heating and cooling.

Table I. $\Delta T_{\mathrm{NI}}$ defined as the difference between

$T_{\mathrm{NI}}$ (center) and $T_{\mathrm{NI}}$ (edge) based on

thickness dependence of $T_{\mathrm{NI}}$ at

(each polymer/5CB) system

\begin{tabular}{lc|lc}
\hline \multicolumn{1}{c|}{ Sample } & $\Delta T_{\mathrm{NI}} / \mathrm{K}$ & \multicolumn{1}{|c}{ Sample } & $\Delta T_{\mathrm{NI}} / \mathrm{K}$ \\
\hline Pdi-iPF & -0.003 & S-PI(AL1051) & 0.015 \\
PCNEt-iPF & 0 & PVC & 0.017 \\
S-PI(DA2) & 0.007 & PVA & 0.031 \\
S-PI(DA1) & 0.010 & PVDF & 0.035 \\
S-PI(DA3) & 0.011 & T-PI & 0.050 \\
\hline
\end{tabular}

nematic LC layer sandwiched between substrates which enhance the order of the LC molecular alignment. Figures 5,6 , and Table I thus indicate that the Pdi-iPF surface may reduce the interfacial alignment order of $5 \mathrm{CB}$ molecules, while PVA, PVC, PVDF, and PIs surfaces enhance it. PCNEt-iPF is neutral with respect to enhancement of the interfacial alignment order of 5CB.

Table II shows the surface free energy of polymer surfaces, $\gamma_{\mathrm{d}}, \gamma_{\mathrm{h}}$, and $\gamma_{\mathrm{s}}$ which correspond to a dispersion component of surface free energy, a hydrogen bonding component and sum of $\gamma_{d}$ and $\gamma_{h}$, respectively, as well as the contact angle of $5 \mathrm{CB}$ with the polymer film surface. Pdi-iPF, PCNEt-iPF, and PVDF had lower surface free energy in comparison with PVA, PVC, and PIs. The surface free energy of 5CB is $32-40 \mathrm{~mJ} \mathrm{~m}^{-2},{ }^{15,16}$ which is close to that of Pdi-iPF, PCNEt-iPF, SPI(DA-2), and less than that of others except PVDF. In the cases of amorphous polymers, $\Delta T_{\mathrm{NI}}$ of $5 \mathrm{CB}$ contacted with the polymer surface with higher surface free energy was larger than for polymer surfaces with lower surface free energy as shown in Table I. 5CB molecules may thus align on the polymeric surface so as to minimize interfacial free energy between LC and polymer surface.

Figures 7 and 8 show plots of $\Delta T_{\mathrm{NI}}$ against the dispersion component, $\gamma_{\mathrm{d}}$ and hydrogen bonding component, $\gamma_{h}$ of the surface free energy, respectively. For amorphous polymers such as Pdi-iPF, PCNEt-iPF, PVC, and S-PIs, it is apparent from Figure 7 that $\Delta T_{\mathrm{NI}}$ linearly increases with $\gamma_{\mathrm{d}}$. No significant relationship between $\Delta T_{\mathrm{NI}}$ and $\gamma_{\mathrm{h}}$ was detected for amorphous polymers as shown in Figure 8. The hydrophobic group or moiety in a polymer chain is preferentially oriented toward the outside from the surface, while the hydrophilic group is so innerwardly to minimize the interfacial free energy between LC molecules and polymer surface, especially in the case of amorphous polymers. In nematic LC the magnitude of surface free energy of LC increases with LC molecular alignment. ${ }^{17,18}$ This indicates that $\gamma_{\mathrm{d}}$ increases with the interfacial order of LC to minimize the difference of dispersion component in surface free energy between amorphous polymer and LC. LC molecular alignment at the (amorphous polymer/LC) interface, in other words, the intermolecular interaction between polymer surface and LC molecules may thus be strongly dependent on the magnitude of dispersion component rather than hydrogen bonding component. The hydrophobic intermolecular interaction at the (polymer/LC) interface would be more responsible for increase in the interfacial molecular alignment order of LC.

There should thus be correlation between $\gamma_{d}$ of polymer and contact angle of LC on the polymer surface. Figures 9 (a) and (b) show plots of $\gamma_{d}$ and $\gamma_{h}$ against the contact angle of $5 \mathrm{CB}$ with each polymer surface. From Figure 9(a) the magnitude of contact angle of 5CB with each polymer surface decreases almost linearly with increase in the dispersion component of surface free energy. However, no systematic correlation between the hydrogen bonding component of polymer surface free energy and contact angle was observed as shown in Figure 9(b). Interactions at the (crystalline polymer/5CB) 
Table II. Surface free energy, aggregation state of each polymer and contact angle of $5 \mathrm{CB}$ with the polymer surface

\begin{tabular}{|c|c|c|c|c|c|}
\hline \multirow{2}{*}{ Sample } & \multicolumn{3}{|c|}{ Surface free energy $/ \mathrm{mJ} \mathrm{m}^{-2}$} & \multirow{2}{*}{$\begin{array}{l}\text { Contact angle of } 5 \mathrm{CB} \\
\text { with each polymer } /^{\circ}\end{array}$} & \multirow{2}{*}{$\begin{array}{l}\text { Aggregation } \\
\text { state }\end{array}$} \\
\hline & $\gamma_{\mathrm{d}}$ & $\gamma_{\mathrm{h}}$ & $\gamma_{\mathrm{s}}$ & & \\
\hline Pdi-iPF & 26.9 & 7.7 & 34.6 & 38 & Amorphous \\
\hline PCNEt-iPF & 30.9 & 8.2 & 39.1 & 27 & Amorphous \\
\hline S-PI(DA-3) & 33.6 & 18.5 & 52.1 & 18 & Amorphous \\
\hline S-PI(DA-2) & 34.1 & 3.0 & 37.1 & 17 & Amorphous \\
\hline S-PI(DA-1) & 37.6 & 11.0 & 48.6 & 7 & Amorphous \\
\hline PVC & 43.9 & 1.6 & 45.5 & 5 & Amorphous \\
\hline S-PI(AL1051) & 44.0 & 7.6 & 51.6 & 2 & Amorphous \\
\hline PVDF & 23.2 & 7.1 & 30.3 & 14 & Crystalline \\
\hline PVA & 37.1 & 7.5 & 44.6 & 2 & Crystalline \\
\hline T-PI & 41.2 & 3.5 & 44.7 & 2 & Crystalline \\
\hline
\end{tabular}

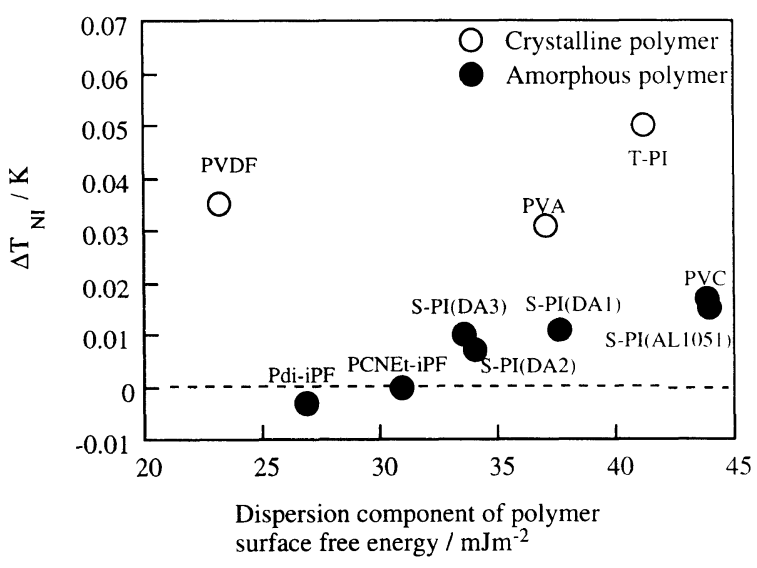

Figure 7. Plots of $\Delta T_{\mathrm{NI}}$ against $\gamma_{\mathrm{d}}$.

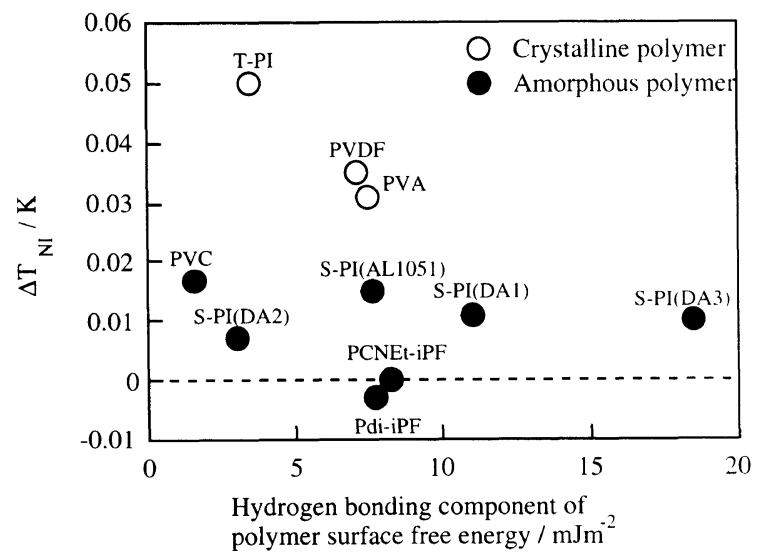

Figure 8. Plots of $\Delta T_{\mathrm{NI}}$ against $\gamma_{\mathrm{h}}$.

interface would thus appear generally stronger than that at the (amorphous polymer/5CB) interface, since the contact angle of $5 \mathrm{CB}$ on the crystalline polymers is smaller than on the amorphous polymers, even though molecular aggregation structure at the crystalline polymer and amorphous polymer surface was not investigated.

Crystalline polymers such as PVA, PVDF, and T-PI showed remarkably large $\Delta T_{\mathrm{NI}}$ in comparison with any other amorphous polymers, as shown in Table I and Figure 7. The surfaces of crystalline polymers such as PVA, PVDF, and T-PI may thus more enhance the interfacial order of $5 \mathrm{CB}$ compared to amorphous polymers such as Pdi-iPF, PCNEt-iPF, PVC, and four

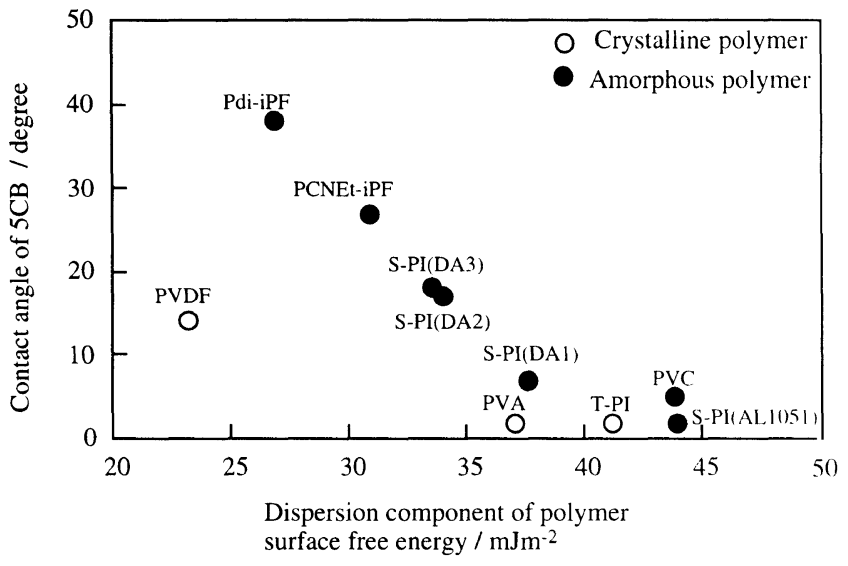

(a)

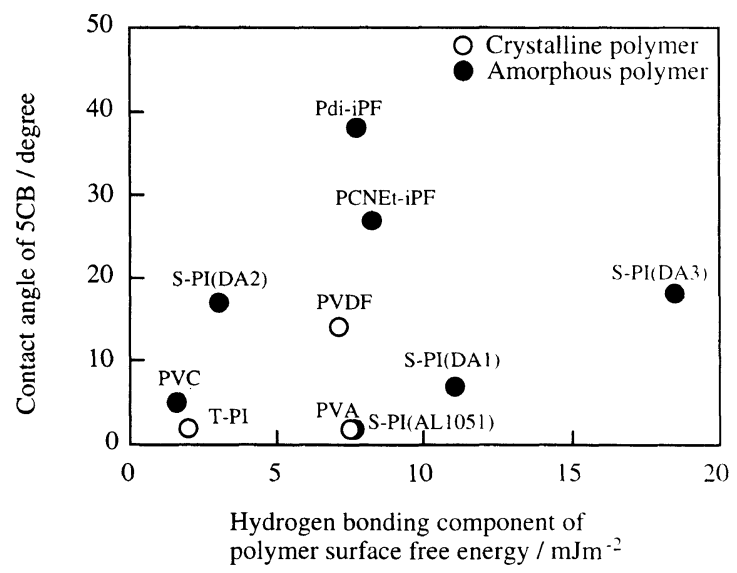

(b)

Figure 9 (a), (b). Plots of $\gamma_{\mathbf{d}}$ and $\gamma_{\mathbf{h}}$ of surface free energy of polymers against contact angle of $5 \mathrm{CB}$ with the polymer.

types of S-PIs.

For an amorphous polymer surface, the alignment order of LC molecules at the interface is mainly determined by intermolecular interactions based on van der Waals force of polymer surface. For a crystalline polymer, the alignment order of the LC molecules at the interface may be affected by structural order of surface polymer chain in addition to the van der Waals force. The surface order of LC at crystalline polymer surface will be discussed in the next paper. 


\section{CONCLUSIONS}

Polymer surfaces with higher surface free energy and crystalline state enhanced the order of LC molecular alignment at the (polymer/LC) interface, in comparison with those with lower surface free energy and an amorphous state. Hydrophobic intermolecular interactions at the (polymer/LC) interface more enhanced interfacial molecular alignment order of LC in comparison with hydrophilic interactions. The order of LC molecular alignment at the interface is thus strongly dependent on surface free energy and aggregation structures on the polymer surface.

\section{REFERENCES}

1. T. Kajiyama, Y. Nagata, S. Washizu, and M. Takayanagi, $J$. Membrane Sci., 11, 39 (1982).

2. T. Kajiyama, A. Miyamoto, H. Kikuchi, and Y. Morimura, Chem. Lett., 813 (1989).
3. A. Miyamoto, H. Kikuchi, Y. Morimura, and T. Kajiyama, New Polym. Mater., 2, 27 (1990).

4. H. Yokoyama, J. Chem. Soc., Faraday Trans. 2, 84, 1023 (1988).

5. P. G. de Gennes, Mol. Cryst. Liq. Cryst., 12, 193 (1971).

6. P. G. de Gennes and J. Prost, "The Physics of Liquid Crystals," 2nd ed, Clarendon Press, Oxford, 1993, Chapter 2.3.

7. A. D. Garbo and M. Nobili, Liquid Crystals, 19, 269 (1995).

8. P. Sheng, Phys. Rev. Lett., 37, 1059 (1976).

9. P. Sheng, Phys. Rev., 26, 1610 (1982).

10. T. J. Sluckin and A. Poniewierski, Phys. Rev. Lett., 55, 2907 (1985).

11. A. Poniewierski and T. J. Sluckin, Liquid Crystals, 2, 281 (1987).

12. S.-K. Hong, H. Kikuchi, and T. Kajiyama, Rep. Prog. Polym. Phys. Jpn., 39, 275 (1996).

13. D. K. Owens and R. C. Wendt, J. Appl. Polym. Sci., 13, 1741 (1969).

14. K. Yamada, M. Takayanagi, and Y. Murata, Polymer, 27, 1054 (1986).

15. T. Uchida, K. Ishikawa, and M. Wada, Mol. Cryst. Liq. Cryst., 60, 37 (1980).

16. J. Cognard, Mol. Cryst. Liq. Cryst., 78 (Suppl. 1) (1982).

17. J. D. Parsons, J. Phys., 37, 1187 (1976).

18. H. Kimura and H. Nakano, J. Phys. Soc. Jpn., 54, 1730 (1985). 